Document downloaded from:

http://hdl.handle.net/10251/35401

This paper must be cited as:

Ibáñez González, JJ.; Hernández García, V. (2011). Solving differential matrix Riccati equations by a piecewise-linearized method based on diagonal Padé approximants. Computer Physics Communications. 182(3):669-678. doi:10.1016/j.cpc.2010.11.024.

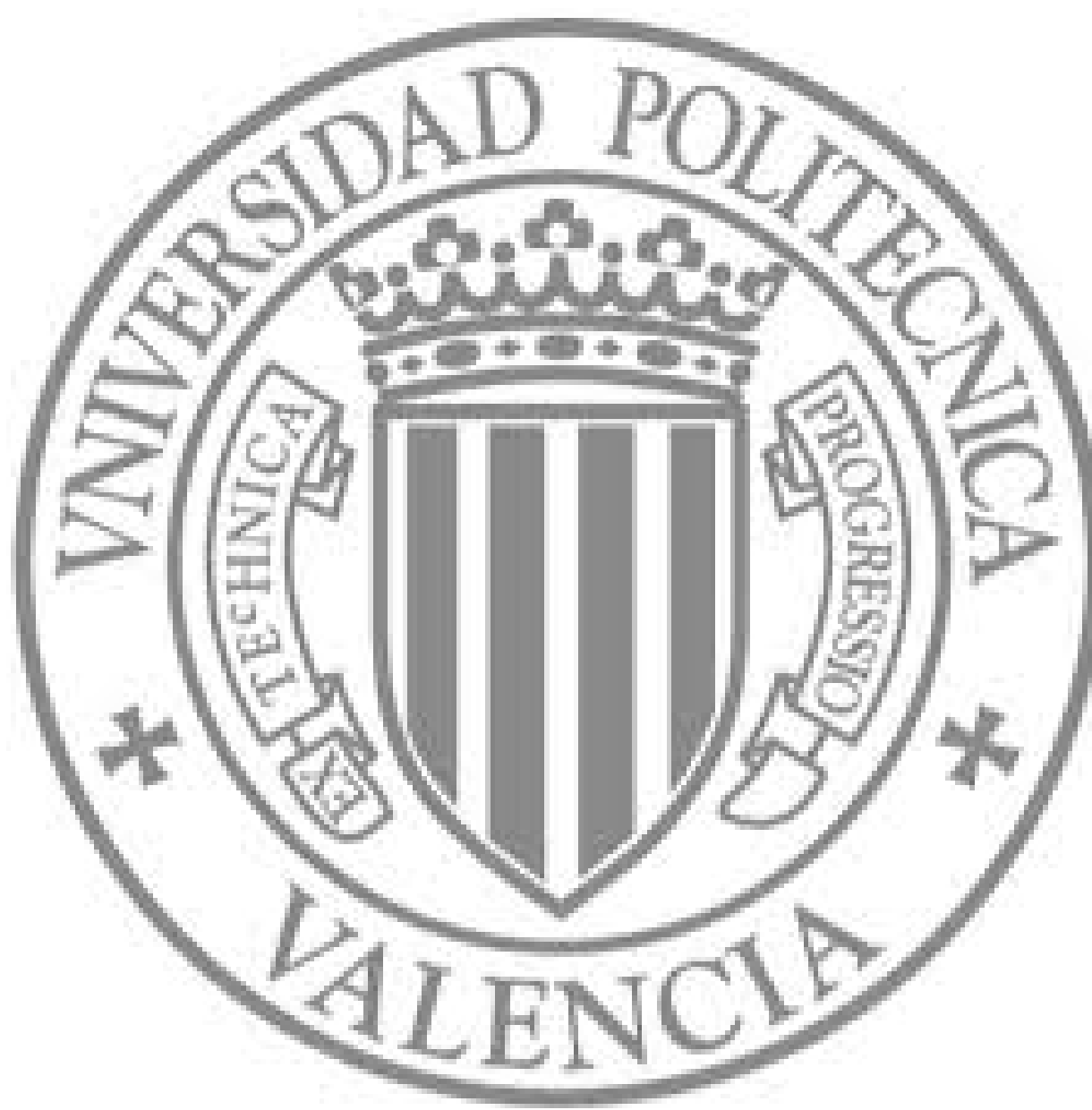

The final publication is available at

http://dx.doi.org/10.1016/j.cpc.2010.11.024

Copyright Elsevier 


\title{
Solving Differential Matrix Riccati Equations by a Piecewise-linearized Method Based on Diagonal Padé Approximants
}

\author{
Javier Ibáñez ${ }^{a, *}$ Vicente Hernández ${ }^{a}$ \\ ${ }^{a}$ Instituto de Instrumentación para Imagen Molecular (I3M), Camino de Vera s/n, \\ 46022-Valencia (Spain)
}

\begin{abstract}
Differential Matrix Riccati Equations (DMREs) appear in several branches of science such as applied Physics and Engineering. For example, these equations play a fundamental role in Control Theory, optimal control, filtering and estimation, decoupling and order reduction, etc. In this paper a new method based on a theorem proved in this paper is described for solving DMREs by a piecewise-linearized approach. This method is applied for developing two block-oriented algorithms based on Diagonal Padé Approximants. MATLAB versions of the above algorithms are developed, comparing, under equal conditions, accuracy and computational costs with other piecewise-linearized algorithms implemented by the authors. Experimental results show the advantages of solving stiff or non-stiff DMREs by the implemented algorithms.
\end{abstract}

Key words: Differential Matrix Riccati Equation (DMRE), Piecewise-linearized Method, Ordinary Differential Equation (ODE), Initial Value Problem (IVP), Linear Differential Equation (LDE), Conmutant Equation, Algebraic Matrix Sylvester Equation (AMSE), Padé Approximants.

PACS: 87.64.Aa

\section{Introduction}

This paper presents a methodology for solving Differential Matrix Riccati Equations (DMREs) based on a piecewise-linearized method which uses Padé

* Corresponding author Javier Ibáñez. Telf: +34-96-3877356. Fax: +34-96-3877359 Email address: jjibanez@dsic.upv.es (Javier Ibáñez ). 
approximants to compute the exponentials of two block-defined matrices. These equations have the form

$$
\begin{gathered}
\dot{X}=A_{21}(t)+A_{22}(t) X-X A_{11}(t)-X A_{12}(t) X, \quad t_{0} \leq t \leq t_{f}, \\
X=X(t) \in \mathbb{R}^{m \times n}, \quad X\left(t_{0}\right)=X_{0} \in \mathbb{R}^{m \times n}
\end{gathered}
$$

where $A_{11}(t) \in \mathbb{R}^{n \times n}, A_{12}(t) \in \mathbb{R}^{n \times m}, A_{21}(t) \in \mathbb{R}^{m \times n}, A_{22}(t) \in \mathbb{R}^{m \times m}$.

DMREs play an important role in the electrodynamic theory of stratified media, in the theory of multimode electric power lines, in the hydraulics of pipe lines, etc. They also appear in Control Theory, for example in the timeinvariant linear quadratic optimal control problem, in the estimation of the system parameters and in the system state, etc.

Since the mid seventies, many different methods have been proposed: linearization methods [1-3], Chandrasekhar method [4], superposition methods [5,6], BDF methods [7-11], Hamiltonian methods [12], unconventional reflexive numerical methods [13], Piecewise-linearized methods [14], etc.

In [14] we developed a piecewise-linearized method based on the Conmutant and we implemented efficient block-oriented algorithms for solving DMREs. In this paper a new piecewise-linearized method for solving DMREs is presented based on Theorem 3 in Section 4, and two block-oriented algorithms based on this method have been developed.

This paper is structured as follows. Section 2 describes a numerical integration of ODEs based on a piecewise-linearized method [15], which has served as the basis for the methods that are described in the following sections. Section 3 describes a piecewise-linearized method developed by the authors [14] which solves DMREs by the Con mutant Equation. Section 4 presents another piecewise-linearized method based on a theorem proved in this paper (Theorem 3) and two block-oriented algorithms. A theoretical study in terms of flops requirements is included. The experimental results of the MATLAB implementations are shown in Section 5. Finally, some conclusions and future work are outlined in Section 6.

\section{Solving ODEs by a Piecewise-linearized Method}

In this section we show a piecewise-linearized method to solve ODEs [15] which is used in Sections 3 and 4. A family of one step methods for solving ODEs are the piecewise-linearized methods $[16,17,15]$. These methods solve an IVP by approximating the right hand-side of the ODE by a degree one Taylor polynomial. The resulting approximation can be integrated analytically to 
obtain the solution in each subinterval and yields the exact solution for linear problems.

Let

$$
\dot{x}(t)=f(t, x(t)), \quad t \in\left[t_{0}, t_{f}\right]
$$

be an ODE with initial value

$$
x\left(t_{0}\right)=x_{0} \in \mathbb{R}^{n},
$$

so that the first order partial derivatives of $f(t, x)$ are continuous on $\left[t_{0}, t_{f}\right] \times$ $\mathbb{R}^{n}$. Given a mesh $t_{0}<t_{1}<\cdots<t_{l-1}<t_{l}=t_{f}$, the ODE (2) can be approximated by a set of Linear Differential Equations (LDEs) obtained as a result of a linear approximation of $f(t, x(t))$ at each subinterval,

$$
\begin{gathered}
\dot{y}(t)=f_{i}+J_{i}\left(y(t)-y_{i}\right)+g_{i}\left(t-t_{i}\right), \quad t \in\left[t_{i}, t_{i+1}\right] \\
y\left(t_{i}\right)=y_{i}, \quad i=0,1, \ldots, l-1,
\end{gathered}
$$

where

$$
\begin{gathered}
f_{i}=f\left(t_{i}, y_{i}\right) \in \mathbb{R}^{n}, \\
J_{i}=\frac{\partial f}{\partial x}\left(t_{i}, y_{i}\right) \in \mathbb{R}^{n \times n}(\text { Jacobian matrix }), \\
g_{i}=\frac{\partial f}{\partial t}\left(t_{i}, y_{i}\right) \in \mathbb{R}^{n} \text { (gradient vector) } .
\end{gathered}
$$

The LDE associated to the first subinterval,

$$
\dot{y}(t)=f_{0}+J_{0}\left(y(t)-y_{0}\right)+g_{0}\left(t-t_{0}\right), \quad t \in\left[t_{0}, t_{1}\right],
$$

is solved considering the initial value $y\left(t_{0}\right)=y_{0}=x_{0}$. Its solution is given by

$$
y(t)=y_{0}+\int_{t_{0}}^{t} e^{J_{0}(t-\tau)}\left[f_{0}+g_{0}\left(\tau-t_{0}\right)\right] d \tau, \quad t \in\left[t_{0}, t_{1}\right] .
$$

Thus, it is possible to compute $y_{1}=y\left(t_{1}\right)$.

By proceeding in the same way, the solution of the LDE associated to the subinterval $i, i=1, \ldots, l-1$, is

$$
y(t)=y_{i}+\int_{t_{i}}^{t} e^{J_{i}(t-\tau)}\left[f_{i}+g_{i}\left(\tau-t_{i}\right)\right] d \tau, \quad t \in\left[t_{i}, t_{i+1}\right]
$$

If $f(t, x)$ is a Lipschitz function on $\left[t_{0}, t_{f}\right] \times \mathbb{R}^{n}$ and its second order partial derivatives are bounded on that region, the above piecewise-linearized method converges [16, pp. 281]. If the $(1,1)$ Padé approximation is used to compute $e^{J_{i}\left(t-t_{i}\right)}$, the above method is consistent of order 2 for autonomous ODEs and 1 for non autonomous ODEs, and linearly stable [18, pp. 26]. 
Theorem 1 ([15]) The solution of the LDE

$$
\begin{aligned}
\dot{y}(t)=f_{i}+J_{i}\left(y(t)-y_{i}\right) & +g_{i}\left(t-t_{i}\right), \quad t \in\left[t_{i}, t_{i+1}\right], \\
y\left(t_{i}\right) & =y_{i}, \\
f_{i} \in \mathbb{R}^{n}, \quad J_{i} & \in \mathbb{R}^{n \times n}, \quad g_{i} \in \mathbb{R}^{n},
\end{aligned}
$$

$i s$

$$
y(t)=y_{i}+E_{12}^{(i)}\left(t-t_{i}\right) f_{i}+E_{13}^{(i)}\left(t-t_{i}\right) g_{i},
$$

where $E_{12}^{(i)}\left(t-t_{i}\right)$ and $E_{13}^{(i)}\left(t-t_{i}\right)$ are blocks $(1,2)$ and $(1,3)$ of $E=e^{C_{i}\left(t-t_{i}\right)}$, where

$$
C_{i}=\left[\begin{array}{ccc}
J_{i} & I_{n} & 0_{n} \\
0_{n} & 0_{n} & I_{n} \\
0_{n} & 0_{n} & 0_{n}
\end{array}\right] .
$$

If $t$ is replaced by $t_{i+1}$ in (4), the approximate solution of the ODE (2) at $t_{i+1}$ is given by

$$
y_{i+1}=y_{i}+E_{12}^{(i)}\left(\Delta t_{i}\right) f_{i}+E_{13}^{(i)}\left(\Delta t_{i}\right) g_{i} .
$$

Therefore, the solutions of the $\operatorname{ODE}(2)$ at $t_{1}, t_{2}, \ldots, t_{l}=t_{f}$ can be computed by using the above expression.

\section{Solving DMREs by a Piecewise-linearized Method based on the Conmutant Equation}

This section describes a piecewise-linearized method developed by the authors [14], which is compared with the piecewise-linearized method developed in this paper. Let us suppose that the right-hand side of (1) ,

$$
F(t, X)=A_{21}(t)+A_{22}(t) X-X A_{11}(t)-X A_{12}(t) X,
$$

is a Lipschitz function on $\left[t_{0}, t_{f}\right] \times \mathbb{R}^{m \times n}$ and its second order partial derivatives are bounded on that region. If vec operator $[19$, p. 244] is applied to DMRE (1), then

$$
\dot{x}(t)=f(t, x(t)), \quad x\left(t_{0}\right)=\operatorname{vec}\left(X_{0}\right),
$$

where

$$
x(t)=\operatorname{vec}(X(t)) \in \mathbb{R}^{m n},
$$

and

$$
f(t, x(t))=\operatorname{vec}\left(A_{21}(t)+A_{22}(t) X(t)-X(t) A_{11}(t)-X(t) A_{12}(t) X(t)\right),
$$


which can be expressed as

$f(t, x(t))=\operatorname{vec}\left(A_{21}(t)\right)+\left[I_{n} \otimes A_{22}(t)-A_{11}^{T}(t) \otimes I_{m}\right] x(t)-\left[I_{n} \otimes\left(X(t) A_{12}(t)\right)\right] x(t)$

or

$f(t, x(t))=\operatorname{vec}\left(A_{21}(t)\right)+\left[I_{n} \otimes A_{22}(t)-A_{11}^{T}(t) \otimes I_{m}\right] x(t)-\left[\left(A_{12}(t) X(t)\right)^{T} \otimes I_{m}\right] x(t)$.

If we consider the mesh $t_{0}<t_{1}<\cdots<t_{l-1}<t_{l}=t_{f}$, and we apply the piecewise-linearized process explained in Section 2, the following LDEs are obtained

$$
\begin{gathered}
\dot{y}(t)=\operatorname{vec}\left(F_{i}\right)+J_{i}\left(y(t)-y_{i}\right)+\operatorname{vec}\left(G_{i}\right)\left(t-t_{i}\right), \quad t \in\left[t_{i}, t_{i+1}\right], \\
y\left(t_{i}\right)=y_{i}, \quad i=0,1, \ldots, l-1,
\end{gathered}
$$

where

$$
\begin{aligned}
F_{i} & =A_{21}\left(t_{i}\right)+A_{22}\left(t_{i}\right) Y_{i}-Y_{i} A_{11}\left(t_{i}\right)-Y_{i} A_{12}\left(t_{i}\right) Y_{i}, \\
G_{i} & =\dot{A}_{21}\left(t_{i}\right)+\dot{A}_{22}\left(t_{i}\right) Y_{i}-Y_{i} \dot{A}_{11}\left(t_{i}\right)-Y_{i} \dot{A}_{12}\left(t_{i}\right) Y_{i} .
\end{aligned}
$$

Since $f(t, x)$ is a Lipschitz function on $\left[t_{0}, t_{f}\right] \times \mathbb{R}^{m n}$ and its second order partial derivatives are bounded on that region, the above piecewise-linearized process converges [16]. If we apply Theorem 1, the solution of (5) at $t_{i+1}$ is

$$
y_{i+1}=y_{i}+E_{12}^{(i)}\left(\Delta t_{i}\right) f_{i}+E_{13}^{(i)}\left(\Delta t_{i}\right) g_{i}
$$

where $E_{12}^{(i)}\left(\Delta t_{i}\right)$ and $E_{13}^{(i)}\left(\Delta t_{i}\right)$ are the $(1,2)$ and $(1,3)$ blocks of matrix $E_{i}=$ $e^{C_{i} \Delta t_{i}}$, where

and

$$
C_{i}=\left[\begin{array}{ccc}
J_{i} & I_{m n} & 0_{m n} \\
0_{m n} & 0_{m n} & I_{m n} \\
0_{m n} & 0_{m n} & 0_{m n}
\end{array}\right],
$$

$$
\begin{gathered}
J_{i}=\frac{\partial f}{\partial x}\left(t_{i}, y_{i}\right)=I_{n} \otimes A_{i}-B_{i}^{T} \otimes I_{m}, \\
A_{i}=A_{22}\left(t_{i}\right)-Y_{i} A_{12}\left(t_{i}\right) \\
B_{i}=A_{11}\left(t_{i}\right)+A_{12}\left(t_{i}\right) Y_{i} .
\end{gathered}
$$

If mat $_{m \times n}$ operator [20, p. 2104] is applied to (6), the approximate solution of (1) at $t_{i+1}$ can be obtained from expression

$$
Y_{i+1}=Y_{i}+\operatorname{mat}_{m \times n}\left(E_{12}^{(i)}\left(\Delta t_{i}\right) \operatorname{vec}\left(F_{i}\right)+E_{13}^{(i)}\left(\Delta t_{i}\right) \operatorname{vec}\left(G_{i}\right)\right) .
$$

Based on the Conmutant Equation, the authors proved in [14] the following theorem and corollary which allow to compute matrix $Y_{i+1}$ in (9). 
Theorem 2 If matrices $A_{i}$ and $B_{i}$ of (8) do not have eigenvalues in common, then the matrix $Y_{i+1}$ in (9) can be computed from

$$
Y_{i+1}=Y_{i}+W_{f}+W_{g}
$$

where $W_{f}, W_{g}$ are the solutions of the Algebraic Matrix Sylvester Equations (AMSEs)

$$
\begin{gathered}
A_{i} W_{f}-W_{f} B_{i}=e^{A_{i} \Delta t_{i}} F_{i} e^{-B_{i} \Delta t_{i}}-F_{i}, \\
A_{i} W_{g}-W_{g} B_{i}=W_{i}-G_{i} \Delta t_{i},
\end{gathered}
$$

and $W_{i}$ satisfies the AMSE

$$
A_{i} W_{i}-W_{i} B_{i}=e^{A_{i} \Delta t_{i}} G_{i} e^{-B_{i} \Delta t_{i}}-G_{i} .
$$

As (11), (12) and (13) have the same coefficient matrices $A_{i}, B_{i}$, the computational cost of computing $Y_{i+1}$ can be reduced.

Corollary 1 If the DMRE (1) is time-invariant and matrices $A_{i}$ and $B_{i}$ of (8) do not have eigenvalues in common, then matrix $Y_{i+1}$ in (9) can be computed as

$$
Y_{i+1}=Y_{i}+W_{f},
$$

where $W_{f}$ is the solution of the AMSE

$$
A_{i} W_{f}-W_{f} B_{i}=e^{A_{i} \Delta t_{i}} F_{i} e^{-B_{i} \Delta t_{i}}-F_{i} .
$$

\section{A Piecewise-linearized Method for Solving DMREs based on Padé Approximants}

As the solution of $\operatorname{LDE}(5)$ associated to subinterval $\left[t_{i}, t_{i+1}\right]$ is

$$
y(t)=y_{i}+\int_{t_{i}}^{t} e^{J_{i}(t-\tau)}\left[\operatorname{vec}\left(F_{i}\right)+\operatorname{vec}\left(G_{i}\right)\left(\tau-t_{i}\right)\right] d \tau, \quad t \in\left[t_{i}, t_{i+1}\right],
$$

then the approximate solution of DMRE (1) at $t_{i+1}$ can be obtained as

$$
Y_{i+1}=\operatorname{mat}_{m \times n}\left(y_{i+1}\right),
$$

where

$$
y_{i+1}=y_{i}+\int_{t_{i}}^{t_{i+1}} e^{J_{i}(t-\tau)}\left[\operatorname{vec}\left(F_{i}\right)+\operatorname{vec}\left(G_{i}\right)\left(\tau-t_{i}\right)\right] d \tau
$$

Theorem 3 The matrix $Y_{i+1}$, which appears in (17), can be computed as follows

$$
Y_{i+1}=Y_{i}+\left[F_{12}^{(i)}\left(\Delta t_{i}\right)+H_{13}^{(i)}\left(\Delta t_{i}\right)\right]\left[F_{22}^{(i)}\left(\Delta t_{i}\right)\right]^{-1},
$$


where $Y_{i}=\operatorname{mat}_{m \times n}\left(y_{i}\right), F_{12}^{(i)}\left(\Delta t_{i}\right)$ and $F_{22}^{(i)}\left(\Delta t_{i}\right)$ are (1,2) and (2,2) blocks of matrix $e^{C_{i} \Delta t_{i}}$,

$$
C_{i}=\left[\begin{array}{cc}
A_{i} & F_{i} \\
0_{n \times m} & B_{i}
\end{array}\right],
$$

and $H_{13}^{(i)}\left(\Delta t_{i}\right)$ is the $(1,3)$ block of matrix $e^{D_{i} \Delta t_{i}}$,

$$
\begin{gathered}
D_{i}=\left[\begin{array}{ccc}
A_{i} & G_{i} & 0_{m \times n} \\
0_{n \times m} & B_{i} & I_{n} \\
0_{n \times m} & 0_{n \times n} & B_{i}
\end{array}\right], \\
F_{i}=A_{21}\left(t_{i}\right)+A_{22}\left(t_{i}\right) Y_{i}-Y_{i} A_{11}\left(t_{i}\right)-Y_{i} A_{12}\left(t_{i}\right) Y_{i}, \\
G_{i}=\dot{A}_{21}\left(t_{i}\right)+\dot{A}_{22}\left(t_{i}\right) Y_{i}-Y_{i} \dot{A}_{11}\left(t_{i}\right)-Y_{i} \dot{A}_{12}\left(t_{i}\right) Y_{i}, \\
A_{i}=A_{22}\left(t_{i}\right)-Y_{i} A_{12}\left(t_{i}\right), \\
B_{i}=A_{11}\left(t_{i}\right)+A_{12}\left(t_{i}\right) Y_{i} .
\end{gathered}
$$

Proof. If we define $s=\tau-t_{i}$ and $\theta=t-t_{i}$, vector $y(t)$ in (16) can be expressed as

$$
\begin{gathered}
y(t)=y_{i}+\int_{0}^{\theta} e^{J_{i}(\theta-s)} \operatorname{vec}\left(F_{i}\right) d s+\int_{0}^{\theta} e^{J_{i}(\theta-s)} g_{i} \operatorname{vec}\left(G_{i}\right) s d s= \\
=y_{i}+\int_{0}^{\theta} e^{\left(I_{n} \otimes A_{i}-B_{i}^{T} \otimes I_{m}\right)(\theta-s)} \operatorname{vec}\left(F_{i}\right) d s+\int_{0}^{\theta} e^{\left(I_{n} \otimes A_{i}-B_{i}^{T} \otimes I_{m}\right)(\theta-s)} \operatorname{vec}\left(G_{i}\right) s d s= \\
=y_{i}+\int_{0}^{\theta}\left(e^{-B_{i}^{T}(\theta-s)} \otimes e^{A_{i}(\theta-s)}\right) \operatorname{vec}\left(F_{i}\right) d s+\int_{0}^{\theta}\left(e^{-B_{i}^{T}(\theta-s)} \otimes e^{A_{i}(\theta-s)}\right) \operatorname{vec}\left(F_{i}\right) s d s .
\end{gathered}
$$

If property (4) of Section 1 is applied, $Y(t)=\operatorname{mat}_{m \times n}(y(t))$ is obtained as

$$
Y(t)=Y_{i}+\int_{0}^{\theta} e^{A_{i}(\theta-s)} F_{i} e^{-B_{i}(\theta-s)} d s+\int_{0}^{\theta} e^{A_{i}(\theta-s)} G_{i} e^{-B_{i}(\theta-s)} s d s,
$$

where

$$
F_{i}=\operatorname{mat}_{m \times n}\left(f_{i}\right), \quad G_{i}=\operatorname{mat}_{m \times n}\left(g_{i}\right) .
$$

In order to compute the first integral in (22), let us consider

$$
C_{i}=\left[\begin{array}{cc}
A_{i} & F_{i} \\
0_{n \times m} & B_{i}
\end{array}\right],
$$


and the exponential of matrix $C_{i} \theta$,

$$
e^{C_{i} \theta}=\left[\begin{array}{cc}
F_{11}^{(i)}(\theta) & F_{12}^{(i)}(\theta) \\
0_{n \times m} & F_{22}^{(i)}(\theta)
\end{array}\right] .
$$

Since

$$
\frac{d e^{C_{i} \theta}}{d \theta}=C_{i} e^{C_{i} \theta}
$$

then

$$
\begin{gathered}
{\left[\begin{array}{cc}
\frac{d F_{11}^{(i)}(\theta)}{d \theta} & \frac{d F_{12}^{(i)}(\theta)}{d \theta} \\
0_{n \times m} & \frac{d F_{22}^{(i)}(\theta)}{d \theta}
\end{array}\right]=\left[\begin{array}{cc}
A_{i} & F_{i} \\
0_{n \times m} & B_{i}
\end{array}\right]\left[\begin{array}{cc}
F_{11}^{(i)}(\theta) & F_{12}^{(i)}(\theta) \\
0_{n \times m} & F_{22}^{(i)}(\theta)
\end{array}\right]} \\
=\left[\begin{array}{cc}
A_{i} F_{11}^{(i)}(\theta) & A_{i} F_{12}^{(i)}(\theta)+F_{i} F_{22}^{(i)}(\theta) \\
0_{n \times m} & B_{i} F_{22}^{(i)}(\theta)
\end{array}\right] .
\end{gathered}
$$

Equating blocks $(1,1),(1,2)$ and $(2,2)$ of both members of the previous equation and considering that

$$
\left.e^{C_{i} \theta}\right|_{\theta=0}=I_{m+n}
$$

the following LDEs are obtained

$$
\begin{gathered}
\frac{d F_{11}^{(i)}(\theta)}{d \theta}=A_{i} F_{11}^{(i)}(\theta), \quad F_{11}^{(i)}(0)=I_{m}, \\
\frac{d F_{22}^{(i)}(\theta)}{d \theta}=B_{i} F_{22}^{(i)}(\theta), \quad F_{22}^{(i)}(0)=I_{n}, \\
\frac{d F_{12}^{(i)}(\theta)}{d \theta}=A_{i} F_{12}^{(i)}(\theta)+F_{i} F_{22}^{(i)}(\theta), \quad F_{12}^{(i)}(0)=0_{m \times n} .
\end{gathered}
$$

Solving (23) and (24), then

$$
\begin{aligned}
& F_{11}^{(i)}(\theta)=e^{A_{i} \theta} \\
& F_{22}^{(i)}(\theta)=e^{B_{i} \theta} .
\end{aligned}
$$

If we replace $F_{22}^{(i)}(\theta)=e^{B_{i} \theta}$ in $(25)$, the following LDE is obtained

$$
\frac{d F_{12}^{(i)}(\theta)}{d \theta}=A_{i} F_{12}^{(i)}(\theta)+F_{i} e^{B_{i} \theta}, \quad F_{12}^{(i)}(0)=0_{m \times n}
$$

therefore

$$
F_{12}^{(i)}(\theta)=\int_{0}^{\theta} e^{A_{i}(\theta-s)} F_{i} e^{B_{i} s} d s
$$


In order to compute the second integral that appears in expression (22), let

$$
D_{i}=\left[\begin{array}{ccc}
A_{i} & G_{i} & 0_{m \times n} \\
0_{n \times m} & B_{i} & I_{n} \\
0_{n \times m} & 0_{n \times n} & B_{i}
\end{array}\right],
$$

and exponential of matrix $D_{i} \theta$,

$$
e^{D_{i} \theta}=\left[\begin{array}{ccc}
H_{11}^{(i)}(\theta) & H_{12}^{(i)}(\theta) & H_{13}^{(i)}(\theta) \\
0_{n \times m} & H_{22}^{(i)}(\theta) & H_{23}^{(i)}(\theta) \\
0_{n \times m} & 0_{n \times n} & H_{33}^{(i)}(\theta)
\end{array}\right] .
$$

Since

then

$$
\frac{d e^{D_{i} \theta}}{d \theta}=D_{i} e^{D_{i} \theta},
$$

$$
\begin{aligned}
& {\left[\begin{array}{ccc}
\frac{d H_{11}^{(i)}(\theta)}{d \theta} & \frac{d H_{12}^{(i)}(\theta)}{d \theta} & \frac{d H_{13}^{(i)}(\theta)}{d \theta} \\
0_{n \times m} & \frac{d H_{22}^{(i)}(\theta)}{d \theta} & \frac{d H_{23}^{(i)}(\theta)}{d \theta} \\
0_{n \times m} & 0_{n \times n} & \frac{d H_{33}^{(i)}(\theta)}{d \theta}
\end{array}\right]} \\
& =\left[\begin{array}{ccc}
A_{i} & G_{i} & 0_{m \times n} \\
0_{n \times m} & B_{i} & I_{n} \\
0_{n \times m} & 0_{n \times n} & B_{i}
\end{array}\right]\left[\begin{array}{ccc}
H_{11}^{(i)}(\theta) & H_{12}^{(i)}(\theta) & H_{13}^{(i)}(\theta) \\
0_{n \times m} & H_{22}^{(i)}(\theta) & H_{23}^{(i)}(\theta) \\
0_{n \times m} & 0_{n \times n} & H_{33}^{(i)}(\theta)
\end{array}\right] \\
& =\left[\begin{array}{ccc}
A_{i} H_{11}^{(i)}(\theta) & A_{i} H_{12}^{(i)}(\theta)+G_{i} H_{22}^{(i)}(\theta) & A_{i} H_{13}^{(i)}(\theta)+G_{i} H_{23}^{(i)}(\theta) \\
0_{n \times m} & B_{i} H_{22}^{(i)}(\theta) & B_{i} H_{23}^{(i)}(\theta)+H_{33}^{(i)}(\theta) \\
0_{n \times m} & 0_{n \times n} & B_{i} H_{33}^{(i)}(\theta)
\end{array}\right] .
\end{aligned}
$$

Equating the corresponding blocks of both members of the previous equation and considering that

the following LDEs can be obtained

$$
\left.e^{D_{i} \theta}\right|_{\theta=0}=I_{m+2 n}
$$

$$
\frac{d H_{11}^{(i)}(\theta)}{d \theta}=A_{i} H_{11}^{(i)}(\theta), \quad H_{11}^{(i)}(0)=I_{m}
$$




$$
\begin{gathered}
\frac{d H_{22}^{(i)}(\theta)}{d \theta}=B_{i} H_{22}^{(i)}(\theta), \quad H_{22}^{(i)}(0)=I_{n}, \\
\frac{d H_{33}^{(i)}(\theta)}{d \theta}=B_{i} H_{33}^{(i)}(\theta), \quad H_{33}^{(i)}(0)=I_{n}, \\
\frac{d H_{12}^{(i)}(\theta)}{d \theta}=A_{i} H_{12}^{(i)}(\theta)+G_{i} H_{22}^{(i)}(\theta), \quad H_{12}^{(i)}(0)=0_{m \times n}, \\
\frac{d H_{23}^{(i)}(\theta)}{d \theta}=B_{i} H_{23}^{(i)}(\theta)+H_{33}^{(i)}(\theta), \quad H_{23}^{(i)}(0)=0_{n \times n}, \\
\frac{d H_{13}^{(i)}(\theta)}{d \theta}=A_{i} H_{13}^{(i)}(\theta)+G_{i} H_{23}^{(i)}(\theta), \quad H_{13}^{(i)}(0)=0_{m \times n} .
\end{gathered}
$$

The solutions of (27), (28) and (29) are

$$
\begin{aligned}
& H_{11}^{(i)}(\theta)=e^{A_{i} \theta}, \\
& H_{22}^{(i)}(\theta)=e^{B_{i} \theta}, \\
& H_{33}^{(i)}(\theta)=e^{B_{i} \theta} .
\end{aligned}
$$

Since $H_{22}^{(i)}(\theta)=e^{B_{i} \theta}$ and $H_{33}^{(i)}(\theta)=e^{B_{i} \theta}$, the solutions of (30) and (31) are

$$
\begin{gathered}
H_{12}^{(i)}(\theta)=\int_{0}^{\theta} e^{A_{i}(\theta-s)} G_{i} e^{B_{i} s} d s \\
H_{23}^{(i)}(\theta)=e^{B_{i} \theta} \theta
\end{gathered}
$$

Finally, replacing $H_{23}^{(i)}(\theta)=e^{B_{i} \theta} \theta$ in (32), the following equation can be obtained

$$
\frac{d H_{13}^{(i)}(\theta)}{d \theta}=A_{i} H_{13}^{(i)}(\theta)+G_{i} e^{B_{i} \theta} \theta, \quad H_{13}^{(i)}(0)=0_{m \times n},
$$

whose solution is

$$
H_{13}^{(i)}(\theta)=\int_{0}^{\theta} e^{A_{i}(\theta-s)} G_{i} e^{B_{i} s} s d s .
$$

Considering the previous expressions and $H_{22}^{(i)}(\theta)=F_{22}^{(i)}(\theta)=e^{B_{i} \theta}$,

$$
Y(t)=Y_{i}+\left[F_{12}^{(i)}(\theta)+H_{13}^{(i)}(\theta)\right]\left[F_{22}^{(i)}(\theta)\right]^{-1} .
$$

If $t$ is replaced by $t_{i+1}$ in the previous expression, we obtain

$$
Y_{i+1}=Y_{i}+\left[F_{12}^{(i)}\left(\Delta t_{i}\right)+H_{13}^{(i)}\left(\Delta t_{i}\right)\right]\left[F_{22}^{(i)}\left(\Delta t_{i}\right)\right]^{-1}
$$

where $\Delta t_{i}=t_{i+1}-t_{i}$. 


\subsection{Algorithm based on Padé approximants with scale-squaring}

The $(s, t)$ Padé approximation to $e^{A}$ is defined by

$$
R_{s t}=\left[D_{s t}(A)\right]^{-1} N_{s t}(A),
$$

where

$$
N_{s t}(A)=\sum_{k=0}^{s} p_{k} A^{k}, \quad p_{k}=\frac{(s+t-k) ! s !}{(s+t) ! k !(s-k) !}
$$

and

$$
D_{s t}(A)=\sum_{k=0}^{s} q_{k} A^{k}, \quad q_{k}=\frac{(-1)^{k}(s+t-k) ! s !}{(s+t) ! k !(s-k) !} .
$$

Non-singularity of $D_{s t}(A)$ is assured if $s$ and $t$ are large enough or if the eigenvalues of $A$ are negative. The problem with this method is that it only provides good approaches near the origin [21, p.573].

Scaling-squaring method is one of the most widely used methods for computing the matrix exponential $[22,23]$ and avoids that problem by exploiting the equality

$$
e^{A}=\left(e^{A / 2^{j}}\right)^{2^{j}}
$$

The idea is to choose $j$ so that $e^{A / 2^{j}}$ can be reliably and efficiently computed, and then to form the matrix $\left(e^{A / 2^{j}}\right)^{2^{j}}$ by repeated squaring. One commonly used criterion for choosing $j$ is to select the smallest natural number such that $\|A\| / 2^{j} \leq 1 / 2[21$, p. 573$]$. There are reasons for favoring the diagonal Padé approximants $(s=t)$, such as for example, their lower computational costs in comparison to the non diagonal Padé approximants.

Algorithm 1 (exmdpa) computes the exponential of a matrix by means of a scaling-squaring diagonal Padé approximation method.

Since diagonal blocks $(1,1)$ and $(2,2)$ of matrices $e^{C_{i} \Delta t}$ and $e^{D_{i} \Delta t}$ are equal, it is possible to obtain a block-oriented algorithm based on Algorithm 1 which allows to compute simultaneously $F_{12}^{(i)}\left(\Delta t_{i}\right)$ and $H_{13}^{(i)}\left(\Delta t_{i}\right)$ without explicitly forming the exponential of matrices $C_{i}$ and $D_{i}$ in expressions (20) and (21). This is done in Algorithm 3. Algorithm 3 (dauvdreplpa) is a double precision auxiliary algorithm that computes the approximate solution at $t_{i+1}$ of time-varying DMRE (1) using a piecewise-linearized method based on Padé approximants. Lines 4-7 of this algorithm avoid overflow problems by controlling that the norms of $\Delta t_{i} C_{i}$ or $\Delta t_{i} D_{i}$ (see expressions (20) and (21)) are lower than a prefixed constant $M$. The approximate computational cost of this 


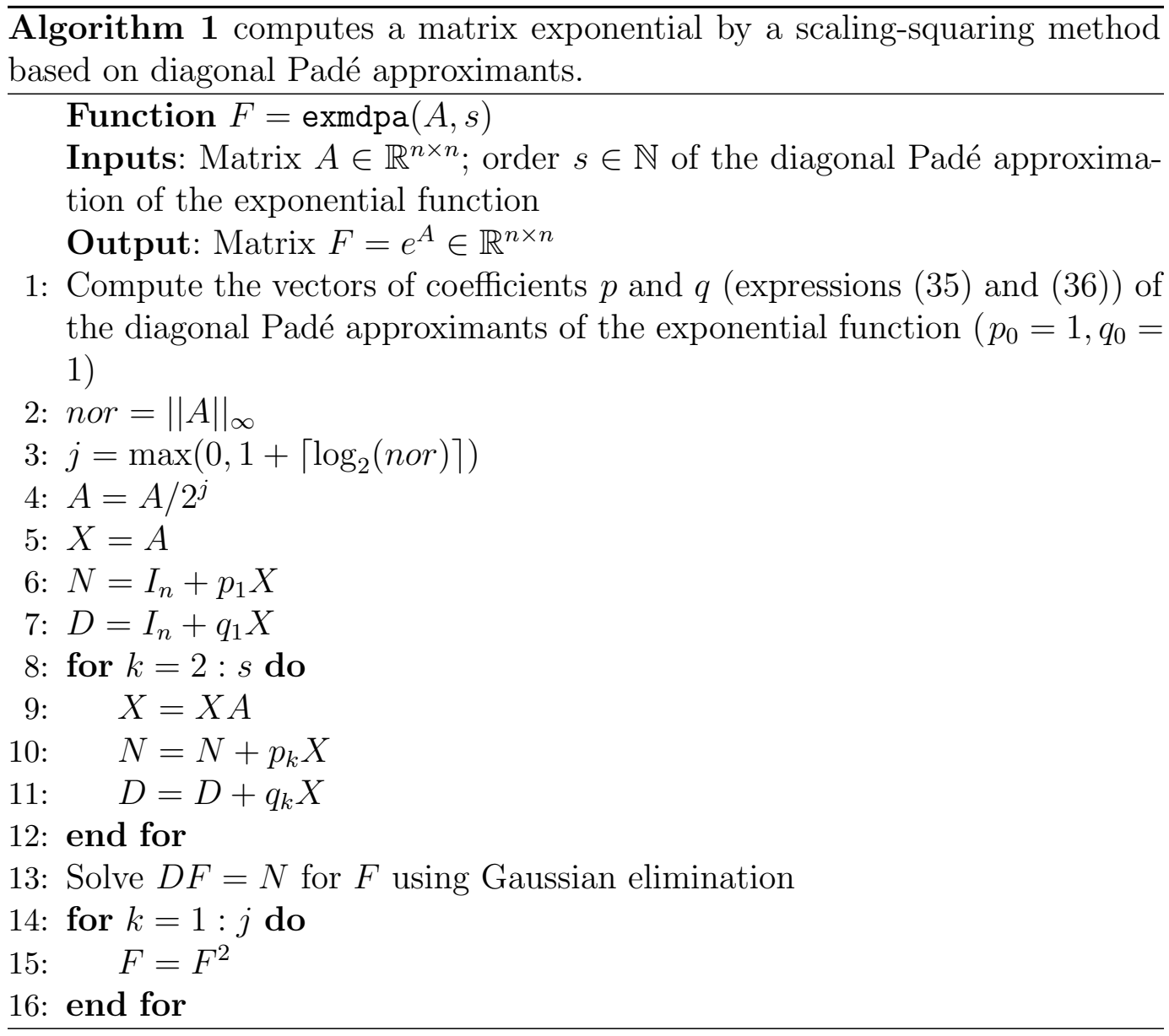

algorithm is $\left(2 m^{3}+6 m^{2} n+6 m n^{2}+4 n^{3}\right) s+\left(2 m^{3}+6 m^{2} n+8 m n^{2}+6 n^{3}\right) j+$ $\frac{2}{3} m^{3}+2 m^{2} n+\frac{10}{3} n^{3}$ flops.

Algorithm 2 (dgevdreplpa) solves, for double precision general matrices, timevarying DMREs by a piecewise-linearized method based on Padé approximants. The approximate cost by iteration of this algorithm is $8 \overline{m^{2}} n+6 m n^{2}+$ $\operatorname{cost}($ data $)+\operatorname{cost}($ datad $)+\operatorname{cost}($ Algorithm 3$)$ flops.

Algorithms 2 and 3 can easily be adapted for time-invariant DMREs : it is sufficient to evaluate the coefficient matrices $A_{i j}$ once, consider $\dot{A}_{22}=0$, and therefore eliminate matrix $G$ from these algorithms. The adapted algorithms for time-invariant will be denoted as dauidreplpa and dgeidreplpa respectively.

\section{$5 \quad$ Numerical experiments}

In this section the algorithms shown in the previous sections are compared with the algorithms presented in [14]. The implementations were tested on 


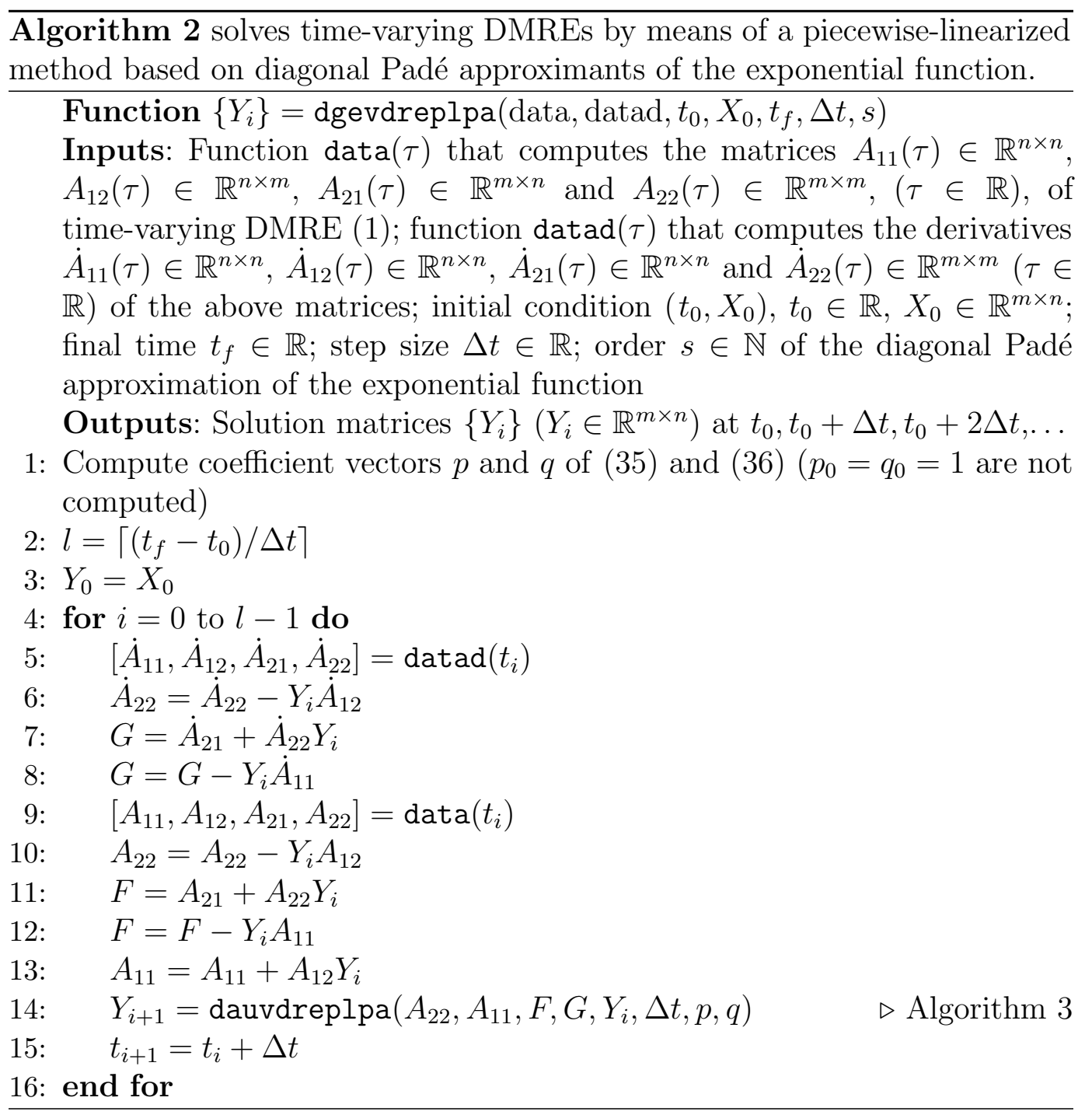

an Intel Core 2 Duo T9400 at $2.52 \mathrm{GHz}$ with 4 GB main memory, using 7.7 (R2008b) MATLAB version. The implemented algorithms are available online at [24].

To test the algorithms a set of six case studies were considered, all with wellknown solutions. For each case study, the values of parameters which offer better accuracy and lower computational cost were determined. Three kinds of tests were carried out varying $\Delta t, t_{f}$ and the dimension of the problem.

In all case studies, the following results are shown:

- Tables which contain the relative error

$$
\operatorname{Er}=\frac{\left\|X-X^{*}\right\|_{\infty}}{\|X\|_{\infty}}
$$

where $X^{*}$ is the computed solution and $X$ is the analytic solution. 


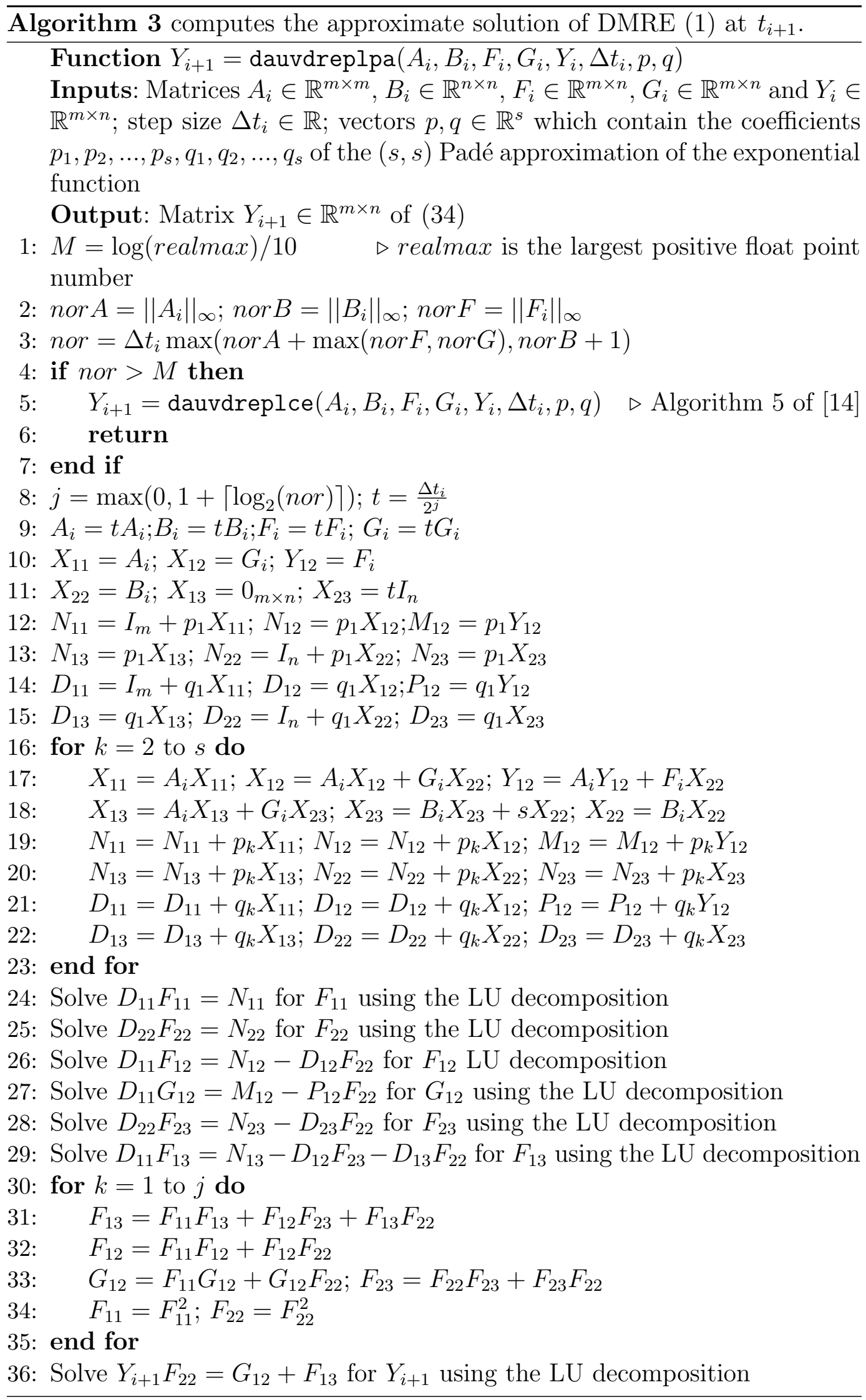


- Tables/graphics with execution times (Te) in seconds.

Below is a short description of compared algorithms:

- dgevdreplpa (Algorithm 2) and dgeidreplpa: They solve time-varying and time-invariant DMREs by the piecewise-linearized method based on the diagonal Padé approximants presented in this paper.

- dgevdreplce (Algorithm 7 of [14]) and dgeidreplce: They solve timevarying and time-invariant DMREs by the piecewise-linearized method based on the Conmutant Equation explained in Section 3.

\subsection{Case Study 1}

The first time-invariant DMRE is taken from a two-point boundary value problem [25]. This DMRE is defined for $t \geq 0$ by the coefficient matrices

$$
\begin{gathered}
A_{11}=\left[\begin{array}{cc}
0 & 0 \\
-100 & -1
\end{array}\right], \quad A_{12}=\left[\begin{array}{cc}
0 & 1 \\
100 & 0
\end{array}\right], \\
A_{21}=\left[\begin{array}{ll}
0 & 1 \\
10 & 0
\end{array}\right], \quad A_{22}=\left[\begin{array}{cc}
0 & 0 \\
-10 & -1
\end{array}\right],
\end{gathered}
$$

and the initial condition

$$
X(0)=\left[\begin{array}{cc}
0 & 0 \\
-1 & 0
\end{array}\right] \text {. }
$$

If $t$ is large, the solution of the previous DMRE is approximately equal to

$$
X=\left[\begin{array}{cc}
1 & 0.11 \\
0 & -0.1
\end{array}\right] \text {. }
$$

In this case study $s=1$ was used for dgeidreplpa and dgeidreplce. In the only test done, final time $t_{f}=30$ was considered and $\Delta t$ was varied between $0.1,0.05$ and 0.01 . Tables 1 and 2 show the results.

Considering the same step size, the conclusions for this case study are:

- Both functions have similar accuracy. 
Table 1

Case study 1: Relative error considering $t_{f}=30$ and $\Delta t$ variable.

\begin{tabular}{cccc}
\hline Er & $\Delta t=0.1$ & $\Delta t=0.05$ & $\Delta t=0.01$ \\
\hline dgeidreplpa & $3.243 \mathrm{e}-14$ & $7.760 \mathrm{e}-15$ & $8.588 \mathrm{e}-16$ \\
\hline dgeidreplce & $3.180 \mathrm{e}-14$ & $6.671 \mathrm{e}-15$ & $8.618 \mathrm{e}-16$ \\
\hline
\end{tabular}

Table 2

Case study 1: Execution time considering $t_{f}=30$ and $\Delta t$ variable.

\begin{tabular}{cccc}
\hline $\mathrm{Te}$ & $\Delta t=0.1$ & $\Delta t=0.05$ & $\Delta t=0.01$ \\
\hline dgeidreplpa & 0.034 & 0.058 & 0.293 \\
\hline dgeidreplce & 0.197 & 0.364 & 1.817 \\
\hline
\end{tabular}

- Relative errors decreased as $\Delta t$ decreased.

- dgeidreplpa has the shorter execution time.

\subsection{Case Study 2}

The second case study [26,7] consists of the following time-invariant DMRE

$$
\dot{X}(t)=A_{21}+A_{22} X(t)-X(t) A_{11}-X(t) A_{12} X(t), \quad 0 \leq t \leq t_{f}
$$

where $A_{11}=0_{n}, A_{12}=A_{21}=\alpha I_{n},(\alpha>0), A_{22}=0_{n}$, and $X_{0} \in \mathbb{R}^{n \times n}$.

The exact solution is given by

$$
X(t)=\left(\alpha\left(X_{0}+I_{n}\right) e^{\alpha t}-\alpha\left(X_{0}-I_{n}\right) e^{-\alpha t}\right)^{-1}\left(\alpha\left(X_{0}+I_{n}\right) e^{\alpha t}+\alpha\left(X_{0}-I_{n}\right) e^{-\alpha t}\right) .
$$

For this case study we considered $s=1$ both for dgeidreplpa and dgeidreplce. Table 3 and Figure 1 show execution times for $\alpha=100$ (stiff problem), $\Delta t=0.1, t_{f}=1$ and the dimension of problem equal to 50, 100, 150 and 200. For two implementations, the relative errors were 0.

Considering the same step size, the conclusions for this case study are:

- Both functions achieved very high accuracy.

- dgeidreplce execution times are longer than dgeidreplpa execution times.

\subsection{Case Study 3}

The third case study [27] consists of the following time-invariant DMRE 
Table 3

Case study 2: Execution time considering $\Delta t=0.1, t_{f}=1$ and $n$ variable.

\begin{tabular}{ccccc}
\hline Te & $n=50$ & $n=100$ & $n=150$ & $n=200$ \\
\hline dgeidreplpa & 0.984 & 3.989 & 8.990 & 16.394 \\
\hline dgeidreplce & 2.941 & 11.709 & 26.293 & 47.282 \\
\hline
\end{tabular}

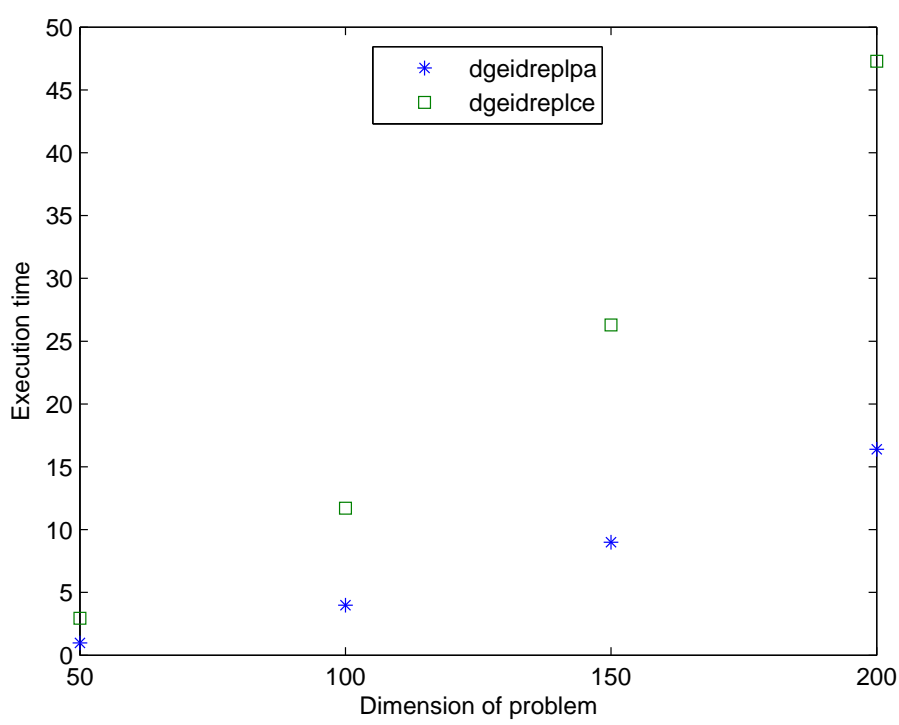

Fig. 1. Case study 2: Execution time considering $\Delta t=0.1, t_{f}=2$ and variable dimension of problem .

$$
\begin{gathered}
\dot{X}(t)=\alpha T_{2^{k}}+T_{2^{k}} X(t)+X(t) T_{2^{k}}-X(t) T_{2^{k}} X(t), \quad t \geq 0, \\
X\left(t_{0}\right)=X_{0},
\end{gathered}
$$

where $X(t), T_{2^{k}} \in \mathbb{R}^{2^{k} \times 2^{k}}$ and $\alpha \in \mathbb{R}^{+}$.

The matrices $T_{2^{k}}$ are generated recursively as follows:

$$
\begin{gathered}
T_{2}=\left[\begin{array}{cc}
-1 & 1 \\
\alpha & 1
\end{array}\right], \\
T_{2^{k}}=\left[\begin{array}{ll}
-T_{2^{k-1}} & T_{2^{k-1}} \\
\alpha T_{2^{k-1}} & T_{2^{k-1}}
\end{array}\right], \quad k \geq 2 .
\end{gathered}
$$

The solution is given by

$$
X(t)=I_{2^{k}}+\frac{(\alpha+1)}{\omega} \tanh (\omega t) T_{2^{k}}
$$


Table 4

Case study 3: Relative error (Er) considering $\Delta t=0.1, t_{f}=5$ and $m=n$ variable.

\begin{tabular}{ccccc}
\hline Er & $n=32$ & $n=64$ & $n=128$ & $n=256$ \\
\hline dgeidreplpa & $1.185 \mathrm{e}-16$ & $1.999 \mathrm{e}-16$ & $3.357 \mathrm{e}-18$ & $7.297 \mathrm{e}-16$ \\
\hline dgeidreplce & $1.185 \mathrm{e}-16$ & $1.999 \mathrm{e}-16$ & $3.357 \mathrm{e}-18$ & $7.297 \mathrm{e}-16$ \\
\hline
\end{tabular}

Table 5

Case study 3: Execution time (Te) considering $\Delta t=0.1, t_{f}=5$ and $m=n$ variable.

\begin{tabular}{ccccc}
\hline Te & $n=32$ & $n=64$ & $n=128$ & $n=256$ \\
\hline dgeidreplpa & 4.894 & 18.221 & 67.976 & 247.246 \\
\hline dgeidreplce & 4.925 & 18.023 & 67.663 & 248.415 \\
\hline
\end{tabular}

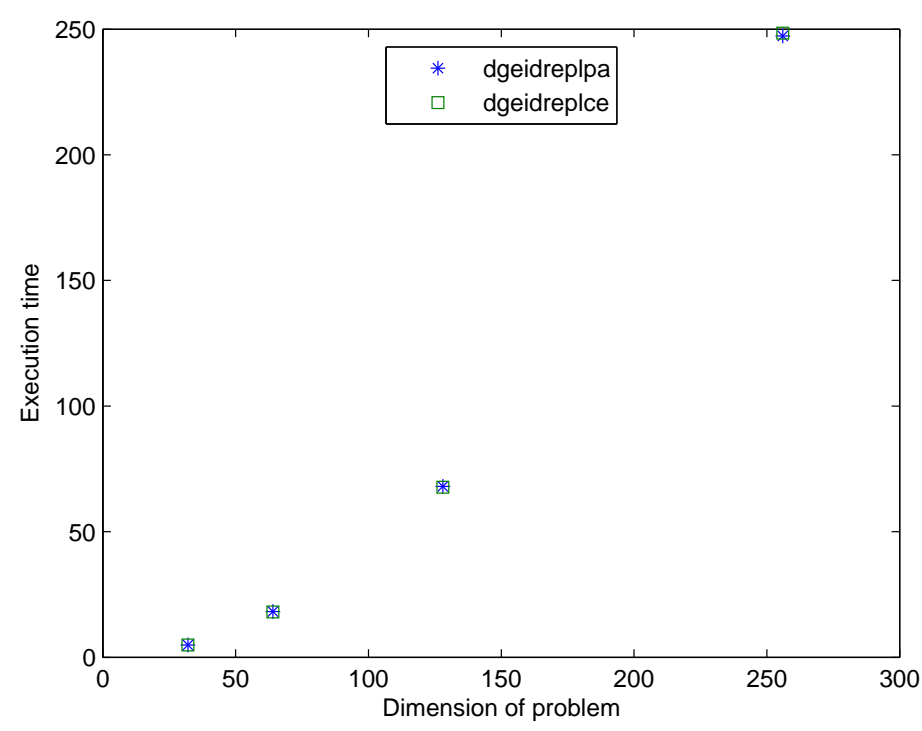

Fig. 2. Case study 3: Execution time considering $t_{f}=5, \Delta t=0.1$ and $n$ variable.

where $\omega=(\alpha+1)^{\frac{k+1}{2}}$.

The parameters of problem were $\alpha=100$ (stiff problem) and $X(0)=I$. The order of Padé approximants for the two implementations was $s=2$. In tests $t_{f}=5$ was considered, varying the dimension of the problem between 32,64 , 128 and 256, and step sizes between 0.1, 0.05, 0.01, 0.005 and 0.001. Both functions achieved smaller relative error for $\Delta t=0.1$. Tables 4 and 5 and Figure 2 show the results for $\Delta t=0.1$. In this case study dgeidreplpa and dgeidreplce achieved very high accuracy for $\Delta t=0.1$ with similar execution times. 
Table 6

Case study 4: Relative error (Er) with $n=1, \Delta t=0.1$ and $t_{f}$ variable.

\begin{tabular}{cccccc}
\hline Er & $t_{f}=10$ & $t_{f}=20$ & $t_{f}=30$ & $t_{f}=40$ & $t_{f}=50$ \\
\hline dgevdreplpa & $4.999 \mathrm{e}-7$ & $2.500 \mathrm{e}-7$ & $1.667 \mathrm{e}-7$ & $1.250 \mathrm{e}-7$ & $1.000 \mathrm{e}-7$ \\
\hline dgevdreplce & $4.999 \mathrm{e}-7$ & $2.500 \mathrm{e}-7$ & $1.667 \mathrm{e}-7$ & $1.250 \mathrm{e}-7$ & $1.000 \mathrm{e}-7$ \\
\hline
\end{tabular}

Table 7

Case study 4: Execution time (Te) with $n=1, \Delta t=0.1$ and $t_{f}$ variable.

\begin{tabular}{cccccc}
\hline Te & $t_{f}=10$ & $t_{f}=20$ & $t_{f}=30$ & $t_{f}=40$ & $t_{f}=50$ \\
\hline dgevdreplpa & 0.063 & 0.121 & 0.178 & 0.235 & 0.293 \\
\hline dgevdreplce & 0.061 & 0.116 & 0.173 & 0.226 & 0.283 \\
\hline
\end{tabular}

\subsection{Case Study 4}

This scalar time-varying DMRE is a widely used for testing stiff problems, known as the "knee problem" ([28,7]), defined as

$$
\dot{x}=1-\frac{t}{\varepsilon} x+\frac{x^{2}}{\varepsilon},-1 \leq t \leq 1, \quad x(-1)=-1, \quad 0<\varepsilon<<1,
$$

associated to the coefficient matrix

$$
A(t)=\left[\begin{array}{ll}
a_{11}(t) & a_{12}(t) \\
a_{21}(t) & a_{22}(t)
\end{array}\right]=\left[\begin{array}{cc}
t / \varepsilon & -1 / \varepsilon \\
0.5 & 0
\end{array}\right], \quad n=m=1 .
$$

The reduced solution $x \cong t$ is stable before 0 and $x \cong 0$ is stable past it.

In the tests done $\varepsilon=10^{-5}$ (stiff problem) and the same order of the diagonal Padé approximants $(s=1)$ was considered. Both functions achieved smaller relative error for $\Delta t=0.1$. Tables 6 and 7 and Figure 3 show relative errors and execution times for $\Delta t=0.1$ and $t_{f}$ variable. In this case study, dgevdreplpa and dgevdreplce achieved smaller relative error for $\Delta t=0.1$, with similar execution times.

\subsection{Case study 5}

This stiff time-varying DMRE [29,7] comes from a stiff two-point boundary value problem. This DMRE is defined as 


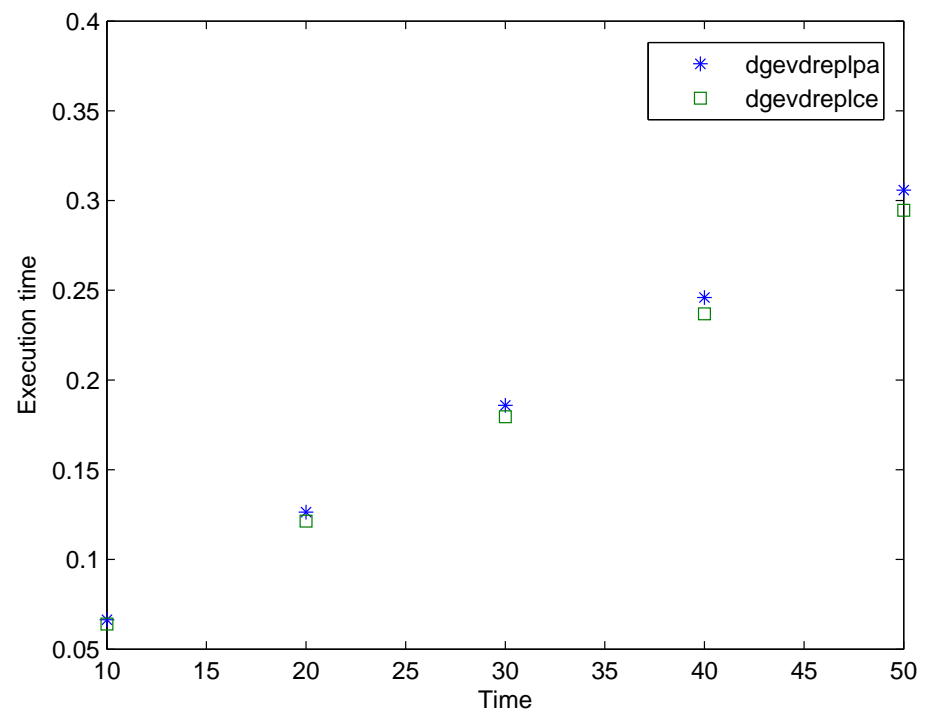

Fig. 3. Case study 4: Execution time considering $\Delta t=0.001$ and $t_{f}$ variable.

$$
\begin{gathered}
A_{11}(t)=\left[\begin{array}{cc}
-t / 2 \varepsilon & 0 \\
0 & 0
\end{array}\right], \quad A_{12}(t)=\left[\begin{array}{cc}
1 / \varepsilon & 0 \\
0 & 1 / \varepsilon
\end{array}\right], \\
A_{21}(t)=\left[\begin{array}{cc}
1 / 2 & 1 \\
0 & 1
\end{array}\right], \quad A_{22}(t)=\left[\begin{array}{cc}
0 & t / 2 \varepsilon \\
0 & 0
\end{array}\right],
\end{gathered}
$$

where $t \geq-1,0<\varepsilon<<1$. The initial condition is

$$
X(-1)=\left[\begin{array}{ll}
0 & 0 \\
0 & 0
\end{array}\right] .
$$

The solution has an initial layer and then it approaches

$$
X(t)=\left[\begin{array}{cc}
-\varepsilon / t & (\sqrt{\varepsilon}+1) /(\sqrt{\varepsilon}-1) \\
0 & \sqrt{\varepsilon}
\end{array}\right] .
$$

For $t$ away from 0 , there is a smooth transition around the origin and then

$$
X(t) \cong\left[\begin{array}{cc}
t / 2 & \sqrt{\varepsilon} \\
0 & \sqrt{\varepsilon}
\end{array}\right]
$$

In the tests $\varepsilon=10^{-5}$ (stiff problem), and the same order of the diagonal Padé 
Table 8

Case study 5: Relative error (Er) with $n=2, \Delta t=0.1$ and $t_{f}$ variable.

\begin{tabular}{cccccc}
\hline Er & $t_{f}=10$ & $t_{f}=20$ & $t_{f}=30$ & $t_{f}=40$ & $t_{f}=50$ \\
\hline dgevdreplpa & $8.668 \mathrm{e}-20$ & $1.776 \mathrm{e}-16$ & $2.891 \mathrm{e}-20$ & $2.168 \mathrm{e}-20$ & $1.421 \mathrm{e}-16$ \\
\hline dgevdreplce & $8.668 \mathrm{e}-20$ & $1.776 \mathrm{e}-16$ & $2.891 \mathrm{e}-20$ & $2.168 \mathrm{e}-20$ & $1.421 \mathrm{e}-16$ \\
\hline
\end{tabular}

Table 9

Case study 5: Execution time (Te) with $n=2, \Delta t=0.1$ and $t_{f}$ variable.

\begin{tabular}{cccccc}
$\mathrm{Te}$ & $t_{f}=10$ & $t_{f}=20$ & $t_{f}=30$ & $t_{f}=40$ & $t_{f}=50$ \\
\hline dgevdreplpa & 0.178 & 0.343 & 0.508 & 0.672 & 0.835 \\
\hline dgevdreplce & 0.177 & 0.342 & 0.502 & 0.667 & 0.829 \\
\hline
\end{tabular}

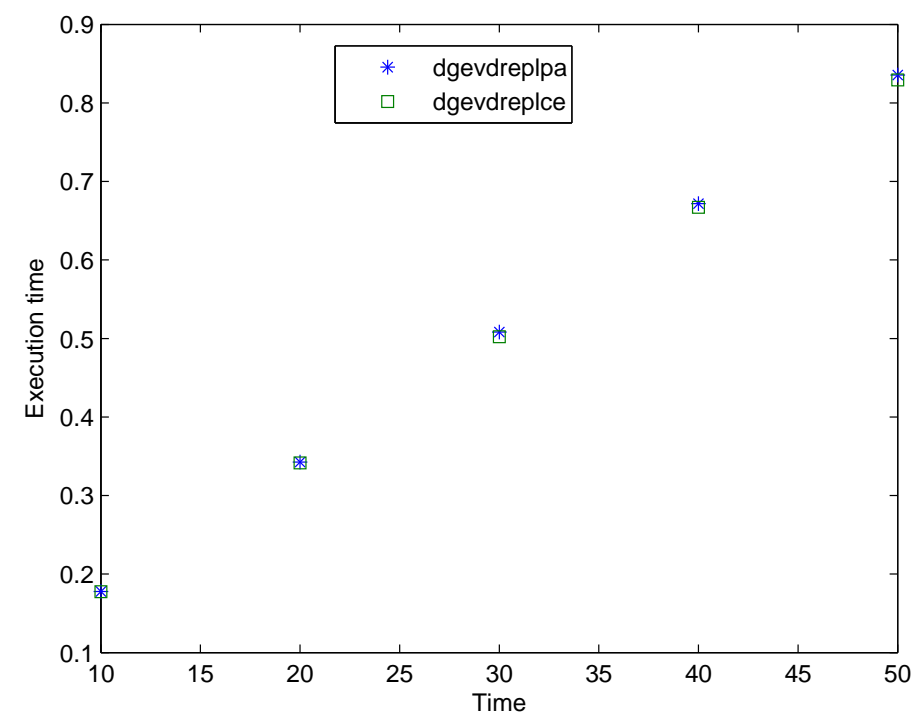

Fig. 4. Case study 5: Execution time considering $\Delta t=0.1$ and $t_{f}$ variable.

approximants $(s=1)$ was considered. In the tests $t_{f}$ was variable with step size $\Delta t=0.1$. Tables 8 and 9 and Figure 4 show that both implementations achieved high accuracy with a similar execution time.

\subsection{Case study 6}

This equation corresponds to a time-varying DMRE [27] defined as

$$
\dot{X}(t)=-X(t) T_{2^{k}}(t)+T_{2^{k}}(t) X(t)-b(t) X^{2}(t)-b(t) I_{2^{k}}, \quad X(0)=I_{2^{k}},
$$

where $X(t) \in \mathbb{R}^{2^{k}}$, and $T_{2^{k}} \in \mathbb{R}^{2^{k}}$ are generated recursively as follows 
Table 10

Case study 6: Relative error (Er) considering $n=8, t_{f}=5$ and $\Delta t$ variable.

\begin{tabular}{cccccc}
\hline Er & $\Delta t=0.1$ & $\Delta t=0.05$ & $\Delta t=0.01$ & $\Delta t=0.005$ & $\Delta t=0.001$ \\
\hline dgevdreplpa & $1.209 \mathrm{e}-02$ & $4.014 \mathrm{e}-03$ & $1.958 \mathrm{e}-04$ & $5.000 \mathrm{e}-05$ & $2.034 \mathrm{e}-06$ \\
\hline dgevdreplce & $1.620 \mathrm{e}-02$ & $4.134 \mathrm{e}-03$ & $1.967 \mathrm{e}-04$ & $5.006 \mathrm{e}-05$ & $2.035 \mathrm{e}-06$ \\
\hline
\end{tabular}

Table 11

Case study 6: Execution time (Te) considering $n=8, t_{f}=5$ and $\Delta t$ variable.

\begin{tabular}{cccccc}
\hline $\mathrm{Te}$ & $\Delta t=0.1$ & $\Delta t=0.05$ & $\Delta t=0.01$ & $\Delta t=0.005$ & $\Delta t=0.001$ \\
\hline dgevdreplpa & 0.021 & 0.043 & 0.228 & 0.418 & 2.092 \\
\hline dgevdreplce & 0.328 & 0.668 & 3.414 & 6.748 & 33.389 \\
\hline
\end{tabular}

Table 12

Case Study 6: Relative error (Er) considering $\Delta t=0.01, t_{f}=5$ and $n$ variable.

\begin{tabular}{ccccc} 
Er & $n=8$ & $n=16$ & $n=32$ & $n=64$ \\
\hline dgevdreplpa & $1.958 \mathrm{e}-04$ & $1.959 \mathrm{e}-04$ & $1.962 \mathrm{e}-04$ & $1.970 \mathrm{e}-04$ \\
\hline dgevdreplce & $1.967 \mathrm{e}-04$ & $1.983 \mathrm{e}-04$ & $2.014 \mathrm{e}-04$ & $2.068 \mathrm{e}-04$ \\
\hline
\end{tabular}

$$
\begin{gathered}
T_{2}=\left[\begin{array}{cc}
a(t) & b(t) \\
-b(t) & a(t)
\end{array}\right], \\
T_{2^{k}}=T_{2} \otimes I_{2^{k-1}}+I_{2} \otimes T_{2^{k-1}}, \quad k \geq 2,
\end{gathered}
$$

where $a(t)=\cos t$ and $b(t)=\sin t$. The analytic solution is

$$
X(t)=\frac{1+\tan (\cos t-1)}{1-\tan (\cos t-1)} I_{2^{k}}
$$

In this case study an order of Padé approximants $s=2$ was selected. Tables 10 and 11 show the results for $n=m=16(k=4), t_{f}=1$ and $\Delta t$ variable. Tables 12 and 13 and Figure 5 show the results for $\Delta t=0.01, t_{f}=5$ and dimension of problem variable. The following conclusions can be emphasized:

- Considering the same step size, both implementations have similar accuracy, but dgevdreplpa has the shorter execution time.

- For both implementations, relative error decreased as $\Delta t$ decreased.

- As dimension of problem is increased dgevdreplce execution time increased quicker than dgevdreplpa execution time: For $n=8$ the execution time ratio is $\frac{3.433}{0.212} \cong 16.193$ and for $n=64$ is $\frac{285.013}{2.381} \cong 119.703$. 
Table 13

Case Study 6: Execution time (Te) considering $\Delta t=0.01, t_{f}=5$ and $n$ variable.

\begin{tabular}{ccccc}
\hline Te & $n=8$ & $n=16$ & $n=32$ & $n=64$ \\
\hline dgevdreplpa & 0.212 & 0.311 & 0.621 & 2.381 \\
\hline dgevdreplce & 3.433 & 19.593 & 54.235 & 285.013 \\
\hline
\end{tabular}

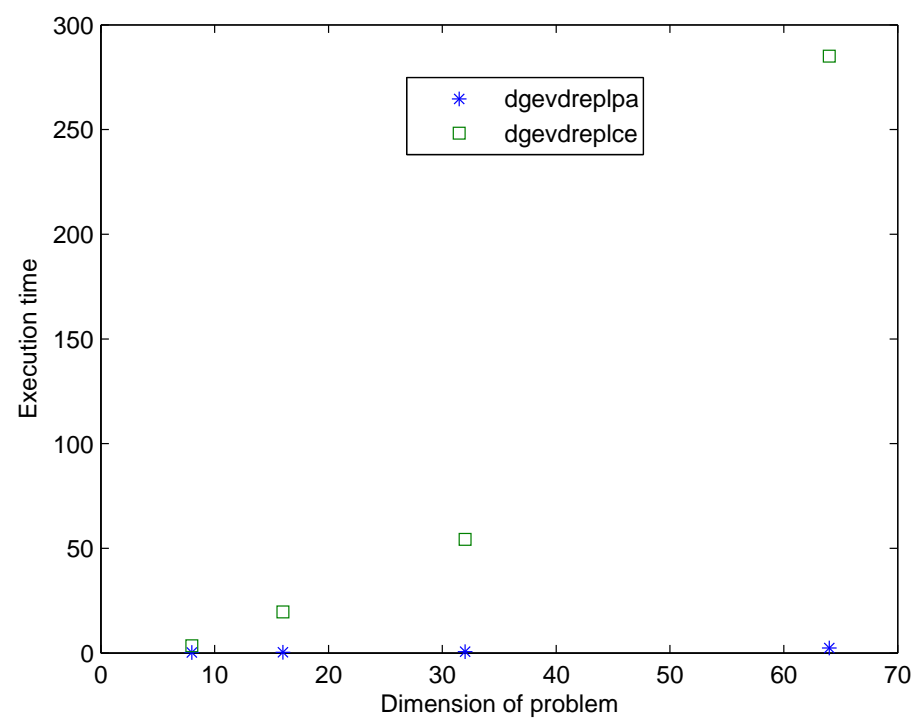

Fig. 5. Case Study 6: Execution time considering $t_{f}=5, \Delta t=0.01$ and variable dimension of problem.

Table 14

Comparison of execution times for the six case studies: the symbols,$+ \cong$ and indicate longer, similar and shorter execution time. The symbols S and NS indicate stiff and non-stiff problem.

\begin{tabular}{ccccccc}
\hline Case Study & 1: NS & $2: \mathrm{S}$ & $3: \mathrm{S}$ & $4: \mathrm{S}$ & $5: \mathrm{S}$ & $6: \mathrm{NS}$ \\
\hline dgeidreplpa-dgevdreplpa & + & + & $\cong$ & $\cong$ & $\cong$ & + \\
\hline dgeidreplce-dgevdreplce & - & - & $\cong$ & $\cong$ & $\cong$ & - \\
\hline
\end{tabular}

\subsection{Summary of results}

Table 14 show a comparison of execution times for the six case studies analyzed, when the implementations have similar accuracy. For each case study, problem stiffness $(\mathrm{S}=$ stiff problem, $\mathrm{NS}=$ non-stiff problem) is indicated.

- In general, for the same step size, the relative errors of all implementations were similar.

- In three case studies, dgeidreplpa-dgevdreplpa execution times were lower than dgeidreplce-dgevdreplce execution times. 
- All implementations showed a good behavior in stiff problems.

\section{Conclusions and future work}

In this paper a method for solving DMREs has been developed. This method is based on Theorem 3 in Section 4 which allows an efficient computation of the integral that appears in the piecewise-linearized methods.

Two MATLAB implementations have been developed based on the piecewiselinearized method developed in Section 4. In order to verify the benefits of these implementations, numerous tests were made on six case studies, comparing, under equal conditions all implementations.

Possible future lines of research are:

- To develop other methods to solve DMREs based on the piecewise-linearized approach. A possibility consists in computing the product of a matrix exponential by a vector using Krylov subspaces (this case will be suitable for higher dimension problems).

- To include adaptive selection of the step size in the algorithms developed in this paper.

- To adapt the implementations for special DMREs such as DMREs with sparse coefficient matrices.

\section{References}

[1] D. R. Vaughan, A negative exponential solution for the matrix Riccati equation, IEEE Trans. on Automatic Control 14 (1) (1969) 72-75.

[2] E. J. Davison, M. C. Maki, The numerical solution of the matrix Riccati differential equation, IEEE Trans. on Automatic Control 18 (1) (1973) 71-73.

[3] C. S. Kenney, R. B. Leipnik, Numerical integration of the differential matrix Riccati equation, IEEE Trans. on Automatic Control 30 (1985) 962-970.

[4] H. Chandrasekhar, Generalized Chandrasekhar algorithms: Time-varying models, IEEE Trans.on Automat. Control 21 (1976) 728-732.

[5] D. W. Rand, P. Winternitz, Nonlinear superposition principles: A new numerical method for solving matrix Riccati equations, Comp. Phys. Commun. 33 (1984) 305-328.

[6] M. Sorine, P. Winternitz, Superposition laws for the solution of differential Riccati equations, IEEE Trans. Automat. Contr. 30 (1985) 266-272. 
[7] L. Dieci, Numerical integration of the differential Riccati equation and some related issues, SIAM Journal on Numerical Analysis. 29 (3) (1992) 781-815.

[8] C. H. Choi, Efficient algorithms for solving stiff matrix-valued Riccati differential equations, Ph.D. thesis, University of California (1988).

[9] P. Benner, H. Mena, BDF methods for large-scale differential Riccati equations, in: Sixteenth International Symposium on Mathematical Theory of Network and Systems (MTNS2004), Katholieke Universiteit Leuven, Belgium, 2004.

[10] E. Arias, V. Hernández, J. Ibáñez, J. Peinado, A fixed point-based BDF method for solving Riccati equations, Applied Mathematics and Computation 188 (2) (2007) 1319-1333.

[11] V. Hernández, J. Ibáñez, E. Arias, J. Peinado, A GMRES-based BDF method for solving differential Riccati equations, Applied Mathematics and Computation 196 (2) (2008) 613-626.

[12] J. M. Sanz-Serna, Symplectic integrators for hamiltonian problems: an overview, Acta Numerica 1 (1992) 243-286.

[13] L. Ren-Cang, Unconventional reflexive numerical methods for matrix differential Riccati, Tech. Rep. 2000-36, Department of Mathematics, University of Kentucky (2000).

[14] J. Ibáñez, V. Hernández, Solving differential matrix Riccati equations by a piecewise-linearized method based on the conmutant equation, Computer Physics Communications 180 (2010) 2103-2114.

[15] J. Ibáñez, V. Hernández, E. Arias, P. Ruiz, Solving initial value problems for ordinary differential equations by two approaches: BDF and piecewise-linearized methods, Computer Physics Communications 180 (5) (2009) 712-723.

[16] J. I. Ramos, C. M. García, Piecewise-linearized methods for initial-value problems, Applied Mathematics and Computation 82 (1997) 273-302.

[17] C. M. García, Piecewise-linearized and linearized $\theta$-methods for ordinary and partial differential equation problems, Computer \& Mathematics with Applications 45 (2003) 351-381.

[18] C. M. García, Métodos de linealización para la resolución numérica de ecuaciones diferenciales, Ph.D. thesis, Departamento de Lenguajes y Ciencias de la Computación, Universidad de Málaga (1998).

[19] R. A. Horn, C. R. Johnson, Topics in Matrix Analysis, Cambridge University Press, London, 1991.

[20] J. Ibáñez, V. Hernández, Solving differential matrix Riccati equations by a piecewise-linearized method based on diagonal Padé approximants, Computer Physics Communications(To appear).

[21] G. H. Golub, C. V. Loan, Matrix Computations, 3rd Edition, Johns Hopkins Studies in Mathematical Sciences, The Johns Hopkins University Press, 1996. 
[22] C. B. Moler, C. V. Loan, Nineteen dubious ways to compute the exponential of a matrix, twenty-five years later*, SIAM Review 45 (2003) 3-49.

[23] N. J. Higham, The scaling and squaring method for the matrix exponential revisited, SIAM J. Matrix Anal. Appl. 26 (4) (2005) 1179-1193.

[24] http://www.grycap.upv.es/dmretoolbox.

[25] S. Pruess, Interpolation schemes for collocation solution of TPBVPs, SIAM Journal on Scientific and Statistical Computing 7 (1986) 322-333.

[26] G. H. Meyer, Initial Value Methods for Boundary Value Problems, Academic Press, New York, 1973.

[27] C. H. Choi, Time-varying Riccati differential equations with known analytic solutions, IEEE Trans. Automat. Contr. 37 (1992) 642-645.

[28] G. Dahlquist, L. Edsberg, G. Sköllermo, G. Söderlind, Are the Numerical Methods and Software Satisfactory for Chemical Kinetics?, in Numerical Integration of DE and Large Linear Systems, Vol. 968/1992 of Lecture Notes in Computer Mathematics, Springer Berlin / Heidelberg, 1982, pp. 149-164.

[29] D. L. Brown, J. Lorenz, A high-order method for stiff boundary value problems with turning points, SIAM Journal on Scientific and Statistical Computing 8 (1987) 790-805. 